Received: 09.06.2018

Revised: 05.09.2018

Accepted: 19.10 .2018

DOI: $10.17804 / 2410-9908.2018 .6 .037-050$

\title{
HYDROGEN DIAGNOSTICS OF METALS AND ALLOYS
}

\author{
A. M. Polyanskiy ${ }^{1, \text { a) }}$, V. A. Polyanskiy ${ }^{2,3, \text { b)* }}$, K. P. Frolova, 2, c), and Yu. A. Yakovleve, 3, d) \\ ${ }^{I}$ RDC Electron \& Beam Technology, Ltd., 6 Bronevaya St., St. Petersburg, 198188, Russian Federation \\ ${ }^{2}$ Peter the Great St. Petersburg Polytechnic University, \\ 29 Politekhnicheskaya, St., St. Petersburg, 195251, Russian Federation \\ ${ }^{3}$ Institute for Problems in Mechanical Engineering, RAS, \\ 61 Bolshoy Ave., V. O., St. Petersburg, 199178, Russian Federation \\ a) iD https://orcid.org/0000-0002-6470-9583 ampol@electronbeamtech.com; \\ b) iD https://orcid.org/0000-0002-1199-1028 vapol@mail.ru; \\ c) iD https://orcid.org/0000-0003-0376-4463 kspfrolova@ gmail.com; \\ d) (iD https://orcid.org/0000-0002-5041-0441 \yura.yakovlev@ gmail.com \\ *Corresponding author. E-mail: vapol@mail.ru \\ Address for correspondence: V. O., Bolshoy pr., 61, St. Petersburg 199178, Russian Federation \\ Tel.: +7 92174806 37; fax: +7 8123214771
}

Within the framework of this paper, we review the development of the problem of hydrogen diagnostics for metals. Metal sample enrichment techniques based on the hydrogen vacuum extraction method had been used for a long time. The development of industrial control technologies has led to the almost complete replacement of vacuum techniques with atmospheric ones. As a result, systematic errors have occurred. These errors lead to multiple differences of certified hydrogen concentration values from measured ones for standard samples.

In this paper, we analyze reasons for the genesis of systematic errors observed for hydrogen measurements while applying the thermal conductivity cell technique. As a result, we have demonstrated that measurements resulting from sample heating and melting in an inert gas flow depend on the heat capacity of the sample and the surface temperature of the melting pot. This explains multiple errors and even negative values in measurements of low hydrogen concentrations.

Keywords: hydrogen diagnostics, hydrogen analyzer, extraction in an inert gas flow, thermal conductivity cell.

\section{Acknowledgment}

The research was supported by the RFBR, projects No. 18-08-00201, 18-31-00329 and 17-08-00783.

\section{References}

1. Gesner G.W. Process of making alloys of iron and hydrogen. US Patent 670,775, 1901.

2. Kinzel A.B. Method of casting steel ingots. US Patent 1,888, 1932.

3. Andrew T., Thomas K.B. Process of manufacturing steel. US Patent 695,264, 1902.

4. $\quad$ Bernhard O. Leitfaden fbr Gießereilaboratorien, Berlin, Heidelberg, Springer, 1915. 44p. ISBN 978-3-662-40626-7. DOI: 10.1007/978-3-662-41106-3.

5. Keiichi O. On the importancy of hydrogen-brittleness as a defect in steel qualities. Tetsu-toHagane, 1938, vol. 24, no. 11, pp. 1005-1013. DOI: 10.2355/tetsutohagane1915.24.11_1005. 
6. De Haas W.J., Hadfield R. On the Effect of the Temperature of Liquid Hydrogen $\left(-252.8{ }^{\circ} \mathrm{C}\right)$ on the Tensile Properties of Forty-One Specimens of Metals Comprising (a) pure iron $99.85 \%$; (b) four carbon steels; (c) thirty alloy steels; (d) copper and nickel; (e) four non-ferrous alloys. Philosophical Transactions of the Royal Society of London. Series A, 1934, vol. 232, pp. 297-332.

7. Zapffe C.A., Sims C.E. Hydrogen embrittlement, internal stress and defects in steel. Trans. AIME, 1941, vol. 145, no. 1941, pp. 225-271.

8. Jordan L., Eckman J.R. Determination of Oxygen and Hydrogen in Metals by Fusion in Vacuum. Industrial \& Engineering Chemistry, 1926, vol. 18, no. 3, pp. 279-282. DOI: $10.1021 / \mathrm{ie} 50195 \mathrm{a} 017$.

9. Brown D. Apparatus for determining hydrogen in steel. US Patent 2,387,878, 1945.

10. Scafe R.M. Determination of Hydrogen in Steel Sampling and Analysis by Vacuum Extraction. Transactions of the American Institute of Mining, Metallurgical and Petroleum Engineers, 1945, vol. 162, p. 375.

11. Olof R. Continuously-operating gas-analyzing apparatus. US Patent 1,644,951, 1927.

12. Willenborg W.J. Method of and means for analyzing gases by differential thermal conductivity measurements. US Patent 2,042,646, 1936.

13. Stewart A.T., Squires G.L. Analysis of ortho-and para-hydrogen mixtures by the thermal conductivity method. Journal of Scientific Instruments, 1955, vol. 32, no. 1, pp. 26.

14. Hulsberg H.A. Hydrogen analyzer. US Patent 2,671,337, 1954.

15. Willenborg W.J. Single cell thermal conductivity measurements. US Patent 2,255,551, 1941.

16. Nolan D., Pitrun M. Diffusible hydrogen testing in Australia. Welding in the World, 2004, vol. 48, no. 1-2, pp. 14-20. DOI: 10.1007/BF03266409.

17. Konopel'ko L.A., Polyanskii A.M., Polyanskii V.A., Yakovlev Y.A. New Metrological Support for Measurements of the Concentration of Hydrogen in Solid Samples. Measurement Techniques, 2018, vol. 60, no. 12, pp. 1222-1227. DOI: 10.1007/s11018-018-1343-3.

18. Hassel A.W., Merzlikin S.V., Mingers A., Georges C., Flock J., Bergers K., Zwettler F. Methodology of Hydrogen Measurements in Coated Steels, Luxembourg, Publications Office of the European Union, 2013, 161 p. ISBN 978-92-79-29712-0.

19. Andronov D.Yu., Arseniev D.G., Polyanskiy A.M., Polyanskiy V.A., Yakovlev Yu.A. Application of multichannel diffusion model to analysis of hydrogen measurements in solid. International Journal of Hydrogen Energy, 2017, vol. 42, no. 1, pp. 699-710. DOI: $10.1016 / 2016.10 .126$.

20. Titov, V.V., Khutoretskii, G.M., Zagorodnaya, G.A. et al. Turbogeneratory. Raschet $i$ konstruktsiya [Turbogenerators: Calculation and Design, N.P. Ivanov and R.A. Lyuter, eds.]. Leningrad, Energiya Publ., 1967.

21. Abarca A.N. High Precision Flow Compensated Thermal Conductivity Detector for Gas Sensing with Read-out Circuit. Master thesis, Delft, 2015.

22. GOST 21132.1-98. Aluminum and aluminum alloys. Methods for determination of hydrogen in solid metal by vacuum hot extraction. (In Russian).

23. Polyanskii A.M., Polyanskii V.A., Yakovlev Yu.A., Study of specimen degassing completeness in analyzing hydrogen content in aluminum alloys. Metallurg, 2011, no. 4, pp. 87-92. (In Russian).

24. Konopelko L.A., Polyanskiy A.M., Polyanskiy V.A., Yakovlev Yu.A. A metrological base for measuring hydrogen concentration for further development of technologies. In: Materialy i tekhnologii dlya Arktiki [Materials and Technologies for the Arctic: international con- 
ference proceedings]. St. Petersburg, 2017, pp. 260-267. ISBN 978-5-900791-36-4. (In Russian).

25. Purcell J.E., Ettre L.S. Analysis of hydrogen with thermal conductivity detectors. Journal of Chromatographic Science, 1965, vol. 3, no. 2, pp. 69-71. DOI: 10.1093/3.2.69.

26. Watanabe M., Inoue R., Ichikawa D., Furusaki K. Development of Thermal Conductivity Type Hydrogen Sensor. ECS Transactions, 2010, vol. 28, no. 20, pp. 31-42. DOI: $10.1149 / 1.3489930$. 
Подана в журнал: 09.06.2018

УДК 539.43:669.018

DOI: $10.17804 / 2410-9908.2018 .6 .037-050$

\title{
ВОДОРОДНАЯ ДИАГНОСТИКА МЕТАЛЛОВ И СПЛАВОВ
}

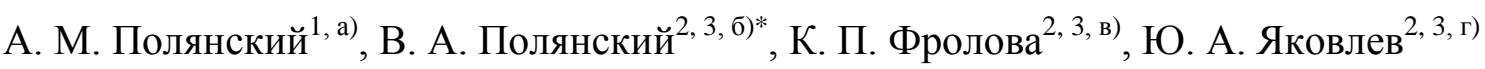 \\ ${ }^{1}$ НПК «Пучковые технологии», ул. Броневая, 6, Санкт-Петербург, Российская Федерация \\ ${ }^{2}$ Институт Проблем Машиноведения РАН, Васильевский остров, \\ Большой проспект, 61, Санкт-Петербург, Российская Федераџия \\ ${ }^{3}$ Санкт-Петербургский Политехнический университет Петра Великого, \\ ул. Политехническая, 29, Санкт-Петербург, Российская Федерация

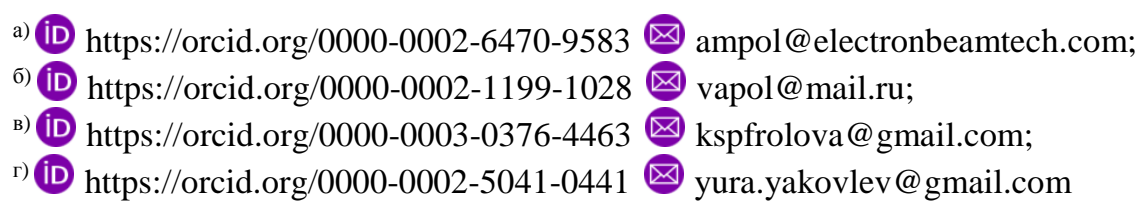
*Ответственный автор. Электронная почта: vapol@mail.ru
Адрес для переписки: Большой проспект, 61, о. Васильевский, 199178, Санкт-Петербург, Российская Федерация Тел.: +7-921-748-06-37; факс: +7-812-321-47-71

В статье дан обзор развития водородной диагностики металлов. Долгое время использовались методы обогащения пробы за счет вакуумной экстракции водорода из металлических образцов. Развитие технологий промышленного контроля привело к практически полному вытеснению вакуумных методик «атмосферными». В результате появились систематические ошибки, дающие многократную разницу между аттестованными и измеренными значениями стандартных образцов при межлабораторных сличениях.

Проведен анализ причин возникновения систематических ошибок, наблюдаемых при измерениях концентрации водорода с помощью ячейки теплопроводности. Показано, что результат измерения приборами, работающими на принципе нагрева и плавления образцов в газе-носителе, зависит не только от стабильности электронных систем и датчиков температуры образца, но и от его теплоемкости, а также состояния поверхности тигля. Этим объясняются многократные систематические ошибки измерения малых концентраций и отрицательные концентрации водорода.

Ключевые слова: водородная диагностика, анализатор водорода, экстракция в потоке газаносителя, ячейка теплопроводности.

\section{1. Введение}

Сильное влияние растворенного водорода на свойства металлов было впервые обнаружено M. Fremy при исследовании влияния газов на свойства сталей в 1861 году. Изучая окклюзию газов металлами, он сделал вывод о том, что сталь поучается из сплава железа только после удаления из него водорода.

Эти знания сорок лет не имели практической ценности. Боле того, водород часто использовался для улучшения качества сталей $[1,2]$. Тем не менее, в начале 20 века было обнаружено, что пузыри в слитках бессемеровской стали заполнены водородом [3], а основной причиной возникновения хрупкости сталей, использованных при производстве аммиака, является водород. Этот вид хрупкости получил название водородной. Для ее профилактики предлагалось выдерживать стальные слитки при температуре $700{ }^{\circ} \mathrm{C}$ в течение нескольких часов.

Другой проблемой начала 20 в. было появление несплошностей проката - флокенов. Первоначально их образование объясняли наличием гидрооксидов железа [4]. Позже (практически через 75 лет после открытий M. Fremy) выяснилось, что такой причиной является 
растворенный в сталях водород [5], который накапливается в жидком металле в мартеновской печи.

В начале 40-х гг. началось применение водородной диагностики в промышленности $[6,7]$. Важной особенностью проводимых серийно исследований являлось то, что критические уровни концентрации или содержания водорода были очень низкими: всего 4 миллионных массовых доли. Подобная точность измерения недостижима для большинства аналитических методов до сих пор.

Вследствие этого широко использовались вакуумные методы обогащения пробы $[8,9,10]$, при которых все газы, выделяющиеся из нагреваемого в вакууме металлического образца, откачивались в калиброванный объем, после чего в нем измерялось давление, а затем производился компонентный анализ газовой смеси с выделением водорода.

Главным недостатком вакуумных методов являлась большая длительность подготовительных работ. Как правило, для исключения фоновых потоков водорода необходимо было откачать всю систему измерительного прибора до высокого вакуума после загрузки образца в вакуумную систему и измерить поправку холостого опыта. Для этого необходимо накапливать фоновый поток водорода в калиброванном объеме, на что требовалось около часа времени. Кроме того, сам процесс вакуумной экстракции мог длиться часами или даже сутками.

Пользуясь опытом быстрых анализаторов кислорода, работающих по принципу восстановительного плавления пробы в потоке газа-носителя и позволяющих проводить измерения за несколько минут, производители измерительного оборудования для промышленности предложили использовать аналогичный метод для измерения концентрации водорода. Высокая температура экстракции позволяла сократить время извлечения водорода, а наличие инертного газа-носителя при атмосферном давлении позволяло избежать вскипания металлической пробы, а также быстро подготовить измерительную систему методом промывания чистым газом-носителем.

Для измерения концентрации водорода в газе-носителе предлагалось использовать разработанную ранее для хроматографов $[11,12]$ ячейку теплопроводности $[13,14,15]$. Принцип действия этого детектора основан на измерении дифференциальной разницы в теплопроводности чистого инертного газа-носителя и газа-носителя, смешанного с водородом, выделившимся из металлического образца при его нагревании.

Сокращение времени анализа водорода от одного часа до десяти минут имело решающее значение для промышленности. Более дешевые и быстрые «атмосферные» анализаторы стали вытеснять вакуумные из заводских лабораторий, и в настоящий момент в мире серийно производится около десятка различных «атмосферных» анализаторов водорода и единственный - вакуумный.

Отсутствие альтернативы в промышленности постепенно привело к тому, что метрологические аттестации, калибровки и научные исследования стали проводиться в основном с применением «атмосферных» анализаторов.

Данное положение дел привело к значительным проблемам. Оказалось, что водород единственный элемент таблицы Менделеева, по которому не проводятся регулярные межлабораторные сличения аттестованных лабораторий. Данные различных лабораторий по измерению концентрации водорода расходятся в несколько раз $[16,17]$, особенно для стандартных образцов с концентрацией водорода мене 1 ppm [18].

В работе [19] исследовано влияние неполного извлечения водорода при использовании метода плавления в потоке газа-носителя для измерения концентрации водорода в алюминиевых сплавах. Тем не менее, большой разброс результатов измерений наблюдается также для сталей [16-18]. По всей видимости, существует неучтенная причина возникновения больших ошибок измерения малых концентраций водорода (менее 1 ppm).

Цель исследования - проведение всестороннего анализа работы детектора водорода. В работе будут изучены причины возникновения систематических ошибок, наблюдаемых при измерениях концентрации водорода с помощью ячейки теплопроводности в анализаторе во- 
дорода, принцип действия которого основан на нагревании и плавлении образца в потоке инертного газа-носителя аргона.

\section{2. Анализ работы детектора водорода}

В настоящий момент в атмосферных анализаторах водорода распространено два основных принципа детектирования. Более старым является классическая ячейка теплопроводности. В некоторых анализаторах используется окисление водорода на ячейке из закиси меди до воды с последующим детектированием воды в газе-носителе инфракрасным датчиком.

Инфракрасный метод имеет большое количество этапов, проблемных, с точки зрения возникновения систематических ошибок измерения. Так, стандартный аргон или азот особой чистоты, который используется в качестве газа-носителя, содержит по техническим условиям около 10 ppm воды, водорода, углеводородов, которые не удается удалить промышленным способом. В этой связи, трудно ожидать стопроцентного удаления этих газов внутри анализатора до обработки водорода на закиси меди, а уровень «полезной» концентрации водорода имеет тот же порядок 5-20 ppm. Для учета возможных ошибок требуется дополнительный экспериментальный анализ наиболее сильно влияющих на них этапов обработки газа-носителя.

Рассмотрим работу более простого детектора - ячейки теплопроводности. Ее схема представлена на рис. 1.

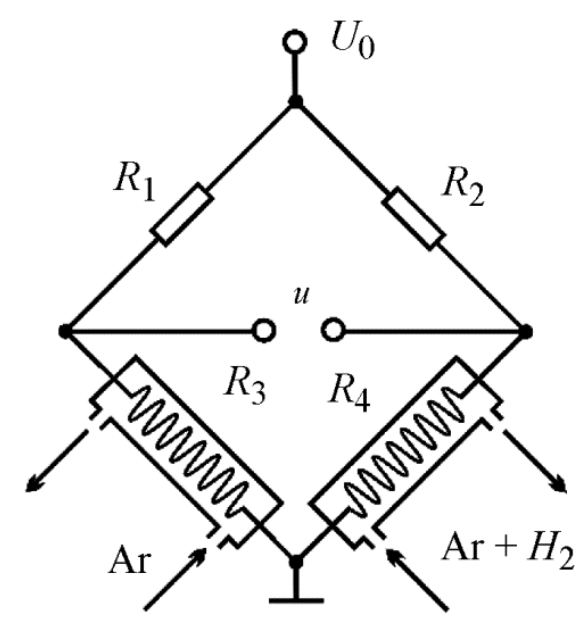

Рис. 1. Схема ячейки теплопроводности

Ячейка теплопроводности представляет собой измерительный мост, на который подается постоянное опорное напряжение $U_{0}$. Ток, величина которого зависит в основном от прецизионных резисторов $R_{1}$ и $R_{2}$, греет платиновые чувствительные резистивные элементы резисторов $R_{3}$ и $R_{4}$ моста. Охлаждение резисторов производиться за счет обдува чистым газом-носителем и тем же газом, который обдувает нагреваемый в особой камере тигель с металлическим образцом и смешивается с водородом.

Прогрев тигля с образцом может производиться как с помощью ВЧ-тока, бесконтактно, так и электрическим током с помощью специального контактного электрода.

Приблизительная схема экстракционной камеры приведена на рис. 2.

После обдува металлического образца в экстракционной камере газ-носитель поступает на очистку от различных веществ, которые выделяются попутно с водородом и могут загрязнить чувствительный платиновый элемент ячейки теплопроводности. В процессе очитки происходит теплообмен газа с порошкообразным сорбентом. Стеклянные трубки с сорбентом размещены на передней панели анализаторов водорода. Считается, что темпера- 
тура газа, смешанного с водородом, выравнивается с температурой опорного газа-носителя, который проходит сквозь такой же сорбент, находящийся в соседней трубке.

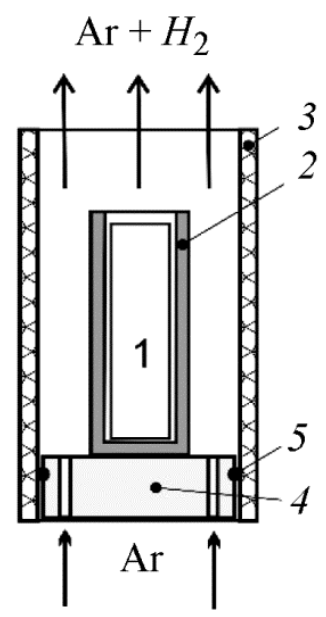

Рис. 2. Схема экстракционной камеры анализатора водорода: 1 - металлический образец; 2 - тигель (как правило, графитовый); 3 - трубка из кварцевого стекла; 4 - заземленный пьедестал для тигля; 5 - уплотнения трубки и пьедестала

После очистки сорбентом газы поступают на детектирующую ячейку теплопроводности (рис. 1), которая фактически измеряет разность потенциалов в диагонали электрометрического моста. Эта разность линейно связана с разностью средних температур платиновых элементов.

Анализаторы водорода фиксируют зависимость разности потенциалов от времени, которая после калибровки является экстракционной кривой. Площадь между экстракционной кривой и фоновой линией равна количеству выделившегося водорода. Для калибровки либо используют стандартный образец, либо вводят водород из калиброванного объема через пористую мембрану в газ-носитель. При этом давление водорода в калиброванном объеме заранее известно.

Можно оценить среднюю разность температур, возникающую при стандартном измерении концентрации водорода. Мощность электрического тока в каждом чувствительном элементе ячейке теплопроводности рассеивается в обдувающий ее газ и вычисляется по формуле (1):

$$
\begin{aligned}
& q_{I A r}=K_{A r} S_{P t}\left(T_{P t A r}-T_{A r}\right) \\
& q_{I \Sigma H_{2}}=K_{\Sigma H_{2}} S_{P t}\left(T_{P t \Sigma H_{2}}-T_{\Sigma H_{2}}\right),
\end{aligned}
$$

где индекс $A r$ относится к характеристикам потока чистого газа-носителя (аргона), индекс $\Sigma \mathrm{H}_{2}$ относится к характеристикам потока смеси газа-носителя с водородом; $q_{I}-$ мощность электрического тока, проходящего через спираль ячейки теплопроводности; $K$ - коэффициент теплоотдачи; $S_{P t}$ - площадь обдуваемой поверхности платинового элемента; $T_{P t}-$ средняя температура платинового элемента; $T$ - температура газа или газовой смеси.

В случае динамического потока газов, ячейка теплопроводности измеряет скорее разность в коэффициенте теплоотдачи от прогреваемого электрическим током платинового чувствительного элемента к обдувающему его газовому потоку, чем теплопроводность в полном смысле этого слова. Этот коэффициент в случае турбулентного газового потока обусловлен, в основном, движением газа в пограничном слое и, как показывает практика, в чистом водороде лишь в полтора раза больше, чем в воздухе [20]. При рабочих концентрациях водорода 
в газе-носителе порядка единиц объемных миллионных долей (vppm), разница будет совсем несущественной. Поэтому внутри ячейки теплопроводности необходимо обеспечить ламинарный поток газа. В этом случае коэффициент теплоотдачи вычисляется по формуле (2):

$$
K=0,5(G r \cdot P r)^{0,25} \frac{\lambda}{d}
$$

где $G r$ - число Грассхофа; $P r$ - число Прандтля; $\lambda$ - коэффициент теплопроводности; $d$ - определяющий размер канала.

Определяющий размер канала определяется по формуле (3):

$$
d=\frac{S}{P},
$$

где $S$ - площадь поперечного сечения канала; $P$ - периметр поперечного сечения канала.

Число Грассхофа и число Прандтля вычисляется по формулам (4) и (5) соответственно:

$$
\begin{gathered}
G r=\frac{g d^{3}}{v^{2}} \frac{\left(T-T_{c p}\right)}{T_{c p}}, \\
\operatorname{Pr}=\frac{\alpha C}{\lambda},
\end{gathered}
$$

где $g$ - ускорение свободного падения; $v$ - коэффициент кинематической вязкости среды; $T-T_{c p}$ - перепад температур между нагреваемым/охлаждаемым объектом и средой; $T_{c p}$ - температура охлаждающего газа; $\alpha$ - коэффициент динамической вязкости газа; $C$ - коэффициент теплоемкости газа.

Коэффициент кинематической вязкости газа вычисляется по формуле (6):

$$
v=\frac{\alpha}{\rho}
$$

где $\rho$ - плотность газа.

При подстановке всех коэффициентов, характеризующих поток и заданных выражениями (3)-(6), в формулу (2) для вычисления коэффициента теплоотдачи, получим следующее выражение (7):

$$
K=0,5 \frac{\lambda}{d}\left(\frac{\alpha C}{\lambda} \frac{g d^{3}}{v^{2}} \frac{1}{T_{c p}}\right)^{0,25}\left(T-T_{c p}\right)^{0,25}=\tilde{k}_{c p}\left(T-T_{c p}\right)^{0,25} .
$$

Для определения разницы температур между спиралью, обдуваемой потоком чистого аргона, и спиралью, обдуваемой потоком смеси аргона с водородом, учтем, что:

$$
q_{I A r}=q_{I \Sigma H_{2}}=q_{I}, T_{A r}=T_{\Sigma H_{2}}=T_{c p} .
$$

Тогда рассеиваемую газом мощность электрического тока в каждом из чувствительных платиновых элементов можно определить по формуле (9), соответственно для $\tilde{k}_{c p}=\tilde{k}_{A r}, T_{P t}=T_{P t A r}$, и $\tilde{k}_{c p}=\tilde{k}_{\Sigma H_{2}}, T_{P t}=T_{P t \Sigma H_{2}}:$ 


$$
q_{I}=\tilde{k}_{c p} S_{P t}\left(T_{P t}-T_{c p}\right)^{1,25}
$$

Выражение для искомой разницы температур между спиралями ячейки теплопроводности имеет следующий вид:

$$
\Delta T=T_{P t A r}-T_{P t \Sigma H_{2}}=\sqrt[1,25]{\frac{q_{I}}{\tilde{k}_{A r} S_{P t}}}-\sqrt[1,25]{\frac{q_{I}}{\tilde{k}_{\Sigma H_{2}} S_{P t}}}=\left(T_{P t A r}-T_{A r}\right)\left[1-\sqrt[1,25]{\frac{\tilde{k}_{A r}}{\tilde{k}_{\Sigma H_{2}}}}\right] .
$$

Поскольку для вычисления разности средних температур между платиновыми чувствительными элементами необходимо определить отношение приведенных коэффициентов теплоотдачи $\tilde{k}_{A r} / \tilde{k}_{\Sigma H_{2}}$, найдем для аргона и смеси аргона с водородом вместо коэффициента $\tilde{k}_{c p}$ коэффициенты $K_{c p}^{*}$, включающие только различающиеся параметры двух газовых сред и выражаемые соотношением (11):

$$
K_{c p}^{*}=\lambda\left(\frac{\alpha C}{\lambda v^{2}}\right)^{0,25}
$$

В качестве рабочих температур газовой среды будем рассматривать значения температур $20^{\circ} \mathrm{C}$ и $600{ }^{\circ} \mathrm{C}$, так как именно в таком диапазоне они варьируются в различных измерительных приборах. Различные характеристики аргона и водорода, вычисленные при температуре среды $20^{\circ} \mathrm{C}$ и $600{ }^{\circ} \mathrm{C}$ представлены в таблицах 1,2 соответственно.

Таблица 1 - Характеристики аргона

\begin{tabular}{|c|c|c|c|c|c|}
\hline$T_{c p},{ }^{\circ} C$ & $\lambda_{A r}, B m / \mu \cdot K$ & $\alpha_{A r} \cdot 10^{-6}, \Pi a \cdot c$ & $C_{A r}$, Дж$/ \kappa 2 \cdot K$ & $\rho_{A r}, \kappa 2 / M^{3}$ & $v_{A r} \cdot 10^{-6}, \mu^{2} / c$ \\
\hline 20 & 0,018 & 22,15 & 521 & 1,78 & 12 \\
\hline 600 & 0,039 & 48,70 & 521 & 0,56 & 87 \\
\hline
\end{tabular}

Таблица 2 - Характеристики водорода

\begin{tabular}{|c|c|c|c|c|c|}
\hline$T_{c p},{ }^{\circ} \mathrm{C}$ & $\lambda_{A r}, \mathrm{Bm} / \mathrm{M} \cdot K$ & $\alpha_{A r} \cdot 10^{-6}, \Pi a \cdot c$ & $C_{A r}$, Дж$/ \kappa 2 \cdot K$ & $\rho_{A r}, \kappa 2 / \mathrm{M}^{3}$ & $\nu_{A r} \cdot 10^{-6}, \mu^{2} / c$ \\
\hline 20 & 0,191 & 8,80 & 14260 & 0,090 & 98 \\
\hline 600 & 0,433 & 18,28 & 14800 & 0,028 & 653 \\
\hline
\end{tabular}

Вычисление максимальной объемной концентрации водорода в газе-носителе можно сделать, исходя из треугольной аппроксимации пика зависимости потока водорода от времени (экстракционной кривой) по формуле (12):

$$
Q_{V H_{2}}=\frac{V_{H_{2}}}{V_{A r}}=\frac{m_{H_{2}} / \rho_{H_{2}}}{W_{A r} t / 2},
$$

где $Q_{V H_{2}}-$ максимальная объемная концентрация водорода в газе-носителе; $V_{H_{2}}, V_{A r}-$ объем газов при нормальных условиях; $m_{H_{2}}$ - масса водорода; $\rho_{H_{2}}-$ плотность водорода; $W_{A r}-$ объемный расход газа-носителя; $t$ - время проведения анализа. 
Масса водорода вычисляется из предположений о массовой концентрации водорода в 0.5 ppm. Считая массу образца стандартной формы и размеров равной $m_{\text {обр }}=5$ г, масса водорода определяется по формуле (13):

$$
m_{\mathrm{H}_{2}}=0,5 \mathrm{ppm} \cdot m_{\text {обр }}
$$

Возьмем следующие значения параметров при нормальных условиях (по данным конкретных измерительных приборов): $W_{A r}=0,26 \frac{\partial \mu^{3}}{\text { мин }}, t=5$ мин. Тогда максимальная объемная концентрация водорода в газе-носителе составит $Q_{v H_{2}}=51,3 v v p n$ и при температуре газа в ячейке теплопроводности $20{ }^{\circ} \mathrm{C}$, и при температуре $600{ }^{\circ} \mathrm{C}$, так как расход задается дозирующими вентилями на входе в систему, в момент, когда газ холодный.

Коэффициент теплоотдачи для смести аргона с водородом будем вычислять, в силу малости концентрации водорода в газовой смеси, по следующей приближенной линеаризованной формуле (14):

$$
K_{\Sigma H_{2}}=Q_{V H_{2}} K_{H_{2}}+\left(1-Q_{V H_{2}}\right) K_{A r}
$$

Рабочая температура платинового элемента является закрытым параметром. Результаты расчета опубликованы только для некоторых конкретных конструкций чувствительного элемента [20]. Очевидно, что чем выше температура, тем больше чувствительность ячейки к концентрации водорода. Возьмем предельные цифры для температуры газовой среды $20{ }^{\circ} \mathrm{C}$ и $600{ }^{\circ} \mathrm{C}$, а для рабочей температуры платинового чувствительного элемента $700{ }^{\circ} \mathrm{C}$ и температуру плавления $-1770{ }^{\circ} \mathrm{C}$.

В табл. 3 представлены значения разности температур двух платиновых элементов $\Delta T$, вычисленные при разных температурах обдувающей их среды и разных температурах элемента, обдуваемого чистым аргоном.

Таблица 3 - Значения разности температур двух платиновых элементов

\begin{tabular}{|c|c|c|}
\hline$T_{A r},{ }^{\circ} \mathrm{C}$ & $T_{P t A r},{ }^{\circ} \mathrm{C}$ & $\Delta T,{ }^{\circ} \mathrm{C}$ \\
\hline 20 & 700 & 0,075 \\
\hline 20 & 1770 & 0,192 \\
\hline 600 & 700 & 0,005 \\
\hline 600 & 1770 & 0,058 \\
\hline
\end{tabular}

\section{3. Обсуждение результатов}

Полученная нами средняя разница в температурах платиновых чувствительных элементов говорит о высокой чувствительности и стабильности работы электронной схемы анализаторов водорода. Она позволяет достоверно измерять разницу порядка 0,07 \% в электрических сопротивлениях. Вместе с тем, есть несколько физических процессов, которые будут вносить ошибку в измерения.

Газ-носитель и газ-носитель с водородом, выделившимся из образца, кондиционируются перед подачей на входы ячейки теплопроводности. Так как внутри анализаторов течет поток газов, кондиционеры являются, по сути, теплообменными аппаратами, а теплообмен между газовой средой и твердыми поверхностями происходит медленно. Как показывают прикидочные расчеты, при разнице температур в несколько градусов теплообмен фактически прекращается, то есть обеспечить кондиционирование газового потока с точностью поддер- 
жания температуры в доли градуса чрезвычайно тяжело. Это может приводить к тому, что исходная разница температур между газами будет превосходить десятые доли градуса, что автоматически меняет среднюю разницу температур платиновых элементов на близкую по значению величину.

Возникает вопрос - откуда может взяться разница в температурах газовых потоков. Очевидно, что образец и тигель, нагретые до высокой температуры, будут подогревать газноситель, протекающий в непосредственном контакте со стенками тигля (рис. 2). Количество поглощенного газом тепла зависит от степени серости стенок тигля и внутренних стенок трубки из кварцевого стекла, которые имеют тенденцию загрязняться брызгами металла и парами веществ, выделяющихся при нагревании образцов до высокой температуры. Оценочный расчет можно сделать, исходя из представлений о теплоемкости тигля, образца и газаносителя, протекающего мимо образца в процессе анализа содержания водорода. Оценка показывает, что даже при условии, что газ-носитель будет отбирать только половину энергии, потраченной на нагрев образца, перегрев газа-носителя составит сотни градусов.

Пустой тигель из-за примерно в два-три раза меньшей теплоемкости нагреет газ соответственно в два-три раза меньше, чем тигель с образцом. В этой связи «холостой опыт», который позволил бы установить фоновые значения водорода, невозможен. Водород содержится даже в дегазированном металле либо внутри, либо на поверхности [22], поэтому изготовить «нулевой» металлический образец с нулевым содержанием водорода даже при уровне концентраций в десятые доли ррт невозможно. Эта точка зрения находит подтверждение как в наших [23], так и в независимых исследованиях [18].

В нашей работе [23] описаны измерения содержания водорода в 54 калибровочных образцах, выточенных из одного стандарта содержания водорода алюминиевого сплава D16. Измерения проводились в сертифицированной заводской лаборатории на поверенном и сертифицированном анализаторе водорода RH402 (производитель LECO). Три измерения дали нулевой результат и одно - отрицательную концентрацию растворенного водорода. С одной стороны, это означает всего лишь 8 \% негодных измерений, которые нужно отбросить. С другой стороны, отрицательная концентрация в протоколе измерений, безусловно, требует объяснений. Наш анализ работы ячейки теплопроводности позволяет дать такие объяснения. «Провал» экстракционной кривой ниже фона может быть вызван более плотным прилеганием конкретного образца к тиглю. Из-за этого процесс теплоотдачи газу будет идти быстрее, и газ прогреется немного больше. Возможно, также, что тигли имеют разные коэффициенты серости. Из-за этого относительные доли излученного и переданного газу носителю тиглем тепла будет разной, а, следовательно, газ по-разному прогреется за счет теплоотдачи с поверхности тигля.

Последний эффект наблюдался независимыми исследователями. В [18] описаны опыты по измерению концентрации водорода в пустых тиглях с помощью, вероятно, прибора Juwe H-mat 221. В отчете не указано точно, каким прибором производились измерения, но среди перечисленных там «атмосферных» анализаторов, только Н-mat 221 обладал необходимой для измерений чувствительностью.

Важно, что все экстракционные кривые в [18] при нагревании пустого тигля становятся отрицательными. Они располагаются ниже исходного фона на разную величину, которая зависит от частоты использования тигля. Таким образом, интеграл от них относительно фона даст отрицательное значение. Этот результат подтверждает наши оценки и предположения.

Разницу между тиглями легко объяснить, если учесть, что тигель прогревается при стандартном измерении примерно до $1000{ }^{\circ} \mathrm{C}$. Его внешние стенки при этом меняют свою структуру и шероховатость, а также покрываются испарениями из образцов. Таким образом, свойства поверхности тигля и доля тепла, уносимая газом, могут существенно меняться от одного измерения к другому.

Имеется еще одно системное соображение, которое объясняет многочисленные проблемы, возникающие при работе «атмосферных» анализаторов водорода. Как уже отмеча- 
лось, относительный порядок измеряемой величины $-10^{-6}$. Причем, ситуация принципиально не меняется при извлечении водорода в газ-носитель, так как масса газа-носителя, используемого при одном анализе, и стандартная масса образца сопоставимы, например 1 и 5 г.

Серийное промышленное измерение с такой точностью без обогащения пробы будет зависеть от стабильности большого количества параметров, начиная от стабильности электрических параметров АЦП, опорных элементов питания, температуры газа-носителя, теплообмена, и заканчивая стабильностью теплоемкости различных образцов из различных сплавов, которая в принципе не может быть одинаковой, так как калибровка осуществляется по стандартным образцам, которых в гос. реестре всего четыре на весь сортамент сталей, алюминиевых и титановых сплавов. Существенное обогащение пробы возможно только в вакуумных методиках измерения или за счет сорбции водорода (хроматографии).

Полученные нами оценки позволяют объяснить значительный разброс, который наблюдается при измерениях концентрации водорода в аттестованных стандартных образцах в различных аттестованных лабораториях [24].

Важно отметить, что ячейка теплопроводности в интересующем нас диапазоне объемных концентраций водорода обладает нелинейной чувствительностью к ним [25, 26]. Это вносит дополнительные ошибки, особенно в случае, когда калибровка анализатора и измерения происходят в разных диапазонах концентраций.

\section{4. Заключение}

Проведен анализ причин возникновения систематических ошибок при измерениях концентрации водорода в металлах, которые могут достигать при использовании современных измерительных приборов семи-восьми раз. Показано, что принцип детектирования водорода, основанный на его большой теплопроводности в газовой фазе, не позволяет избежать влияния множества факторов окружающей среды, состава пробы, состояния тиглей и условий проведения измерений на его результат. Показано, что это влияние может быть определяющим и существенно менять измеряемую величину концентрации водорода, особенно при концентрации водорода менее 1 ррm.

Данные концентрации находятся за пределами чувствительности большинства современных аналитических методов определения компонентного состава вещества. Их измерения требуют обогащения пробы, которого в атмосферных анализаторах водорода не происходит.

Таким образом, вывод фундаментального отчета европейской программы [18] о том, что не существует надежных стандартных образцов с аттестованным содержанием водорода менее 1 ppm необходимо дополнить выводом о непригодности атмосферных анализаторов водорода для измерений в образцах металлов с концентрацией водорода менее 1 ppm.

\section{Благодарность}

Исследование выполнено при поддержке РФФИ, проекты No. 18-08-00201, 18-3100329 и 17-08-00783.

\section{Литература}

1. Process of making alloys of iron and hydrogen : pat. 670,775 U.S. / Gesner G. W. Publ. 26.03.1901.

2. Method of casting steel ingots : pat. 1,888,132 U.S. / Kinzel A. B. - Publ. 15.11.1932.

3. Process of manufacturing steel : pat. 695,264 U.S. / Andrew T., Thomas K. B. Publ. 11.03.1902.

4. Bernhard O. Leitfaden for GieЯereilaboratorien. - Berlin, Heidelberg : Springer, 1915. 44 p. - ISBN 978-3-662-40626-7. - DOI: 10.1007/978-3-662-41106-3. 
5. Keiichi O. On the importancy of hydrogen-brittleness as a defect in steel qualities // Tetsuto-Hagane. - 1938. - Vol. 24, no. 11 - P. 1005-1013. - DOI: 10.2355/1915.24.11_1005.

6. De Haas W. J., Hadfield R. On the Effect of the Temperature of Liquid Hydrogen $\left(-252.8^{\circ} \mathrm{C}\right)$ on the Tensile Properties of Forty-One Specimens of Metals Comprising (a) pure iron 99.85\%; (b) four carbon steels; (c) thirty alloy steels; (d) copper and nickel; (e) four non-ferrous alloys // Philosophical Transactions of the Royal Society of London. Series A. - 1934. - Vol. 232. P. 297-332.

7. Zapffe C. A., Sims C. E. Hydrogen embrittlement, internal stress and defects in steel // Trans. AIME. - 1941. - Vol. 145, no. 1941. - P. 225-271.

8. Jordan L., Eckman J. R. Determination of Oxygen and Hydrogen in Metals by Fusion in Vacuum // Industrial \& Engineering Chemistry. - 1926. - Vol. 18, no. 3. - P. 279-282. DOI: $10.1021 / \mathrm{ie} 50195 \mathrm{a} 017$.

9. Apparatus for determining hydrogen in steel : pat. 2,387,878 U.S. / Brown W. D. Publ. 30.10.1945.

10. Scafe R. M. Determination of Hydrogen in Steel Sampling and Analysis by Vacuum Extraction // Transactions of the American Institute of Mining, Metallurgical and Petroleum Engineers. 1945. - Vol. 162. - P. 375.

11. Continuously-operating gas-analyzing apparatus : pat. 1,644,951 U.S. / Olof R. Publ. 11.10.1927.

12. Method of and means for analyzing gases by differential thermal conductivity measurements : pat. 2,042,646 U.S. / Willenborg W. J. - Publ. 2.07.1936.

13. Stewart A. T., Squires G. L. Analysis of ortho-and para-hydrogen mixtures by the thermal conductivity method // Journal of Scientific Instruments. - 1955. - Vol. 32, no. 1. - P. 26.

14. Hydrogen analyzer : pat. 2,671,337 U.S. / Hulsberg H. A. - Publ. 9.03.1954.

15. Single cell thermal conductivity measurements : pat 2,255,551 U.S. / Willenborg W. J. Publ. 9.09.1941.

16. Nolan D., Pitrun M. Diffusible hydrogen testing in Australia // Welding in the World. 2004. - Vol. 48, no. 1-2. - P. 14-20. - DOI: 10.1007/BF03266409.

17. New Metrological Support for Measurements of the Concentration of Hydrogen in Solid Samples / L. A. Konopel'ko, A. M. Polyanskii, V. A. Polyanskii, Y. A. Yakovlev // Measurement Techniques. - 2018. - Vol. 60, no. 12. - P. 1222-1227. - DOI: 10.1007/s11018-018-1343-3.

18. Methodology of Hydrogen Measurements in Coated Steels / A. W. Hassel, S. V. Merzlikin, A. Mingers, C. Georges, J. Flock, K. Bergers, F. Zwettler. - Luxembourg : Publications Office of the European Union, 2013 - 161 p. - ISBN 978-92-79-29712-0.

19. Application of multichannel diffusion model to analysis of hydrogen measurements in solid / D. Yu. Andronov, D. G. Arseniev, A. M. Polyanskiy, V. A. Polyanskiy, Yu. A. Yakovlev // International Journal of Hydrogen Energy. - 2017. - Vol. 42, no. 1. - P. 699-710. - DOI: 10.1016/2016.10.126.

20. Турбогенераторы: расчет и конструкция / В. В. Титов, Г. М. Хуторецкий, Г. А. Загородная, Г. П. Вартаньян, Д. И. Заславский, И. А. Смотров / под ред. Н. П. Иванова, Р. А. Лютера. Л. : Энергия, 1967.

21. Abarca A. N. High Precision Flow Compensated Thermal Conductivity Detector for Gas Sensing with Read-out Circuit : master thesis. - Delft, 2015 - 97 p.

22. ГОСТ 21132.1-98. Межгосударственный стандарт. Алюминий и сплавы алюминиевые. Метод определения водорода в твердом металле вакуум-нагревом.

23. Полянский А. М., Полянский В. А., Яковлев Ю. А. Исследование полноты дегазации образцов при анализе содержания водорода в алюминиевых сплавах // Металлург. - 2011. № 4. - С. 87-92.

24. Метрологическая база измерений концентрации водорода для дальнейшего развития технологий : доклады международной конференции «Материалы и технологии для Арктики» / Л. А. Конопелько, А. М. Полянский, В. А. Полянский, Ю. А. Яковлев. - Санкт-Петербург : 
НИЦ «Курчатовский институт», ЦНИИ КМ «Прометей», 2017. - С. 260-267. - ISBN 978-5900791-36-4.

25. Purcell J. E., Ettre L. S. Analysis of hydrogen with thermal conductivity detectors // Journal of Chromatographic Science. - 1965. - Vol. 3, no. 2. - P. 69-71. - DOI: 10.1093/3.2.69.

26. Development of Thermal Conductivity Type Hydrogen Sensor / M. Watanabe, R. Inoue, D. Ichikawa, K. Furusaki // ECS Transactions. - 2010. - Vol. 28, no. 20. - P. 31-42. DOI: $10.1149 / 1.3489930$. 